gerichtet sein und müssen Maßnahmen der Verbreitungsarbeit auch eine Entwicklung des Rechts in den Blick nehmen. Ein solches Verständnis muss umso mehr für die Komponenten der Rotkreuz- und Rothalbmondbewegung gelten, deren »Mission « es ist, »menschliches Leiden überall und jederzeit $z u$ verhüten und $z u$ lindern $\varkappa^{64}$. Wenn eine Organisation wie die Internationale Rotkreuz- und Rothalbmondbewegung es sich zum Ziel setzt, »der Würde des Menschen Achtung zu verschaffen, vor allem in Zeiten bewaffneter Konflikte « ${ }^{65}$,

64 Präambel der Statuten der Bewegung.

65 Ebenda. dann kann keine ihrer Komponenten sich in der Verbreitungsarbeit darauf beschränken, den Inhalt des bestehenden humanitären Völkerrechts »in die Welt« zu tragen. Wenn es der Bewegung ernst ist damit, ihre Grundsätze und Ideale Wirklichkeit werden zu lassen, dann kann sie sich nicht damit begnügen, den Bestand des humanitären Völkerrechts zu bewahren, zu verteidigen und bekannt zu machen. Vielmehr muss sie, wann immer und wo immer sich die Möglichkeit dazu bietet, eine Fortentwicklung des Schutzes des Menschlichkeit in bewaffneten Auseinandersetzungen aktiv und >proaktiv< betreiben.

\title{
Verletzungen des humanitären Völkerrechts im aktuellen Kriegsgeschehen: Ein Überblick
}

\author{
Manuel Probst*
}

\begin{abstract}
International humanitarian law is highly developed and extensively codified but its application is almost exclusively limited to international conflicts, i.e. armed clashes between state armies. Written law dealing with non-international armed conflicts, i.e. conflicts in which at least one party is a non-conventional armed group, can only be found in Article 3 of the four Geneva Conventions and in the Additional Protocol II. Intended to protect civilians in wars Article 3 in particular specifies binding rules for regular and irregular combatants. Since all current wars are of a non-international nature we must ask to what extent this very basic article of international humanitarian law is violated during them.
\end{abstract}

Keywords: Humanitäres Völkerrecht, Kriegsgeschehen, Menschenrechte

\section{Das humanitäre Völkerrecht}

$\mathrm{D}$ as humanitäre Völkerrecht wurde geschaffen, um zwischenstaatliche, symmetrische Kriege zu reglementieren und um anhand dieser Regeln das in Kriegen entstehende Leid zu minimieren. Inzwischen legt das Völkerrecht für alle Staaten ein allgemeines Gewaltverbot fest, welches nur zur Selbstverteidigung bei einem Angriff durch andere Staaten gebrochen werden darf. Diese Form der Reglementierung des Kriegsgeschehens hat jedoch seit 1945 immer mehr an Bedeutung verloren, da sich das Kriegsgeschehen selbst gravierend verändert hat. Ein zwischenstaatlicher Krieg mit regulären Streitkräften auf beiden Seiten ist inzwischen eine absolute Ausnahme geworden. Die Verlagerung des Kriegsgeschehens hin zu innerstaatlichen, asymmetrischen Kriegen, in denen maximal eine reguläre Armee irregulären Streitkräften ge-

\footnotetext{
* Manuel Probst (M.A.) ist Mitarbeiter der Forschungsstelle Kriege, Rüstung und Entwicklung (FKRE) und der Arbeitsgemeinschaft Kriegsursachenforschung (AKUF) der Universität Hamburg.
}

genüber steht, ist der Regelfall geworden. Und selbst in den zwischenstaatlichen Kriegen stehen sich immer seltener zwei reguläre Streitkräfte gegenüber. Es handelt sich beispielsweise weder bei der Hisbollah noch bei den muslimischen Milizen in Somalia um reguläre Armeen. Sie fallen beide nicht unter die im humanitären Völkerrecht festgelegten Bestimmungen für reguläre Streitkräfte. Momentan wird auf der gesamten Erde nicht ein einziger Krieg geführt, für den das humanitäre Völkerrecht eigentlich geschaffen wurde. ${ }^{1}$

Seitdem das Kriegsgeschehen von innerstaatlichen Konflikten geprägt wird, hat es immer wieder Versuche und Initiativen gegeben, diese durch eine Erweiterung oder Abwandlung des humanitären Völkerrechts ebenfalls zu verregeln. Besonders bemerkenswert ist in diesem Zusammenhang sicherlich das zweite Zusatzprotokoll zu den Genfer Konventionen von

\footnotetext{
1 Man könnte vielleicht noch argumentieren, dass es sich z.B. bei den Taliban um (ehemals) staatliche Kräfte handelt, die wiederum gegen andere staatliche Kräfte kämpfen.
} 
1977. In diesem Zusatzprotokoll wird u.a. allen nationalen Befreiungsbewegungen in Dekolonialisierungskriegen der Status einer regulären Streitmacht zugesprochen. ${ }^{2}$ Diese Form der Kriegsführung spielt jedoch im heutigen Kriegsgeschehen keine Rolle mehr. Welche anwendbaren Regeln im humanitären Völkerrecht schränken aber den Handlungsspielraum der Kombattanten in einem nicht-internationalen Krieg ein? Das humanitäre Völkerrecht kommt ideengeschichtlich dem Versuch gleich, einen Mindeststandard an Menschlichkeit auch in dem extremen Fall eines Krieges einzuhalten. Somit gilt, unabhängig von jedweder positiv gesatzten Rechtsordnung, dass die Menschenrechte auch jeden Kombattanten auf Einhaltung eines Mindestmaßes an Menschenrechten besonders gegenüber Unbeteiligten festlegen. ${ }^{3}$ Einen standfesten Rechtspositivisten wird ein solch abstraktes Argument über die universelle Gültigkeit der Menschenrechte kaum beeindrucken. Doch gibt es auch positive Rechtsordnungen innerhalb des humanitären Völkerrechts, die die Handlungsspielräume der Streitkräfte einschränken. In Art. 3, der in allen vier Genfer Konventionen enthalten ist, wird für nicht-internationale Konflikte u.a. festgelegt, dass jede der am Konflikt beteiligten Parteien Folgendes einzuhalten hat: ${ }^{4}$

Personen, die nicht direkt an den Feindseligkeiten teilnehmen, sollen unter allen Umständen mit Menschlichkeit behandelt werden. $\mathrm{Zu}$ diesem Zwecke sind verboten:

(a) Angriffe auf Leib und Leben, namentlich Mord jeglicher Art, Verstümmelung, grausame Behandlung und Folterung (b) Geiselnahme

(c) Beeinträchtigung der persönlichen Würde, namentlich erniedrigende und entwürdigende Behandlung

(d)Verurteilungen und Hinrichtungen ohne vorhergehendes Urteil eines ordnungsgemäß bestellten Gerichtes.

Zusätzlich müssen natürlich Sanktionsmaßnahmen bei Rechtsbruch durch die eigenen Kombattanten geschaffen werden. Die Anwendung der vorstehenden Bestimmungen hat dabei auf den Rechtsstatus der am Konflikt beteiligten Parteien keinen Einfluss und Gefangene müssen somit nicht als Kriegsgefangene behandelt werden.

Sicherlich ist jedoch der nichtstaatliche Akteur in nicht-internationalen Konflikten keine eigene Vertragspartei und man könnte somit behaupten, dass die dargelegten Bestimmungen für ihn deshalb nicht bindend seien. Müsste sich die staatliche Seite somit an Regeln halten, die nichtstaatliche jedoch nicht? Ich bin der Überzeugung, dass die vom Staat abgeschlossenen Verträge für die gesamte Bevölkerung Gültigkeit haben und ihnen somit nicht separat zugestimmt werden muss. Damit würden nichtstaatliche Akteure durch eine Ausnahme von der gewohnheitsrechtlichen Regel, dass Verträge Dritte nicht binden können, dennoch an diese Verträge gebunden wer-

2 Zusätzlich definiert das Zusatzprotokoll auch genauer, was ein nicht-internationaler Konflikt ist. Vgl. McCoubrey, Hilaire: International Humanitarian Law, Ashgate 1998, S. 253ff.

3 Dies hier weiter auszuführen würde sicherlich zu weit führen. Für meine Position zu diesem Thema vgl. Probst, Manuel: Die humanitäre Interventionspflicht, Hamburg 2006.

4 Für eine ausführliche Darstellung des 3. Artikels siehe z.B. McCoubrey, Hilaire: International Humanitarian Law, Ashgate 1998, S. $260 \mathrm{ff}$. den. ${ }^{5}$ Eine sicherlich nicht gänzlich kontraintuitive Sichtweise, denn schließlich fragt ein Rechtsstaat auch nicht jeden einzelnen Bürger, ob er die geltenden Gesetze akzeptieren möchte. Auch wenn dieser Lösungsansatz nicht unbedingt alle zufrieden stellen wird, ist die Alternative, nichtstaatliche Akteure nicht an den 3. Artikel zu binden, noch unbefriedigender. ${ }^{6}$ Im Folgenden werde ich demnach nichtstaatliche Akteure so beurteilen, als ob sie zweifelsfrei an den Art. 3 gebunden wären.

Wie verhält es sich aber mit diesem, in Art. 3 festgelegten, minimalen Standard der Menschlichkeit im aktuellen Kriegsgeschehen? Ziel der vorliegenden Untersuchung ist es, den Grad der Verletzung des Art. 3 in den jeweiligen Kriegen des aktuellen Kriegsgeschehens darzustellen. Der Leser wird dadurch einen Überblick über das aktuelle Kriegsgeschehen und den jeweiligen Verletzungen des humanitären Völkerrechts bekommen. Dabei wird es mir ausschließlich um die jeweiligen Verletzungen des Art. 3 gehen.

\section{Das aktuelle Kriegsgeschehen}

Um das aktuelle Kriegsgeschehen auf die jeweiligen Verletzungen des 3. Artikels zu überprüfen, stellen sich zunächst zwei fundamentale Fragen: Ab wann ist ein Krieg noch Teil des aktuellen Kriegsgeschehens? Und noch viel zentraler: Welche Definition soll man zugrunde legen, um Kriege von anderen Formen der bewaffneten Auseinandersetzung zu unterscheiden?

Um die erste Frage zu beantworten, habe ich mich entschlossen, all diejenigen Kriege als aktuell zu betrachten, die teilweise oder gänzlich in den vergangenen zwölf Monaten ausgetragen wurden. Diese Festlegung auf die letzten zwölf Monate seit März 2006 entbehrt nicht einer gewissen Willkür, ist aber zur Umsetzung des hier dargelegten Vorhabens wichtig. Bei welcher Form von bewaffneten Auseinandersetzungen handelt es sich aber um Kriege? Verschiedene Forschungseinrichtungen und Forscher haben Krieg unterschiedlich definiert. Eine ausführliche Diskussion der verschiedenen Ansätze würde hier sicherlich zu weit führen. In Anlehnung an den ungarischen Friedensforscher István Kende (1917-1988) und fast analog zur Definition der Arbeitsgemeinschaft Kriegsursachenforschung $(\mathrm{AKUF})^{7}$ verstehe ich Krieg als einen gewaltsamen Massenkonflikt, der alle folgenden Merkmale aufweist:

(a) An den Kämpfen sind zwei oder mehr bewaffnete Streitkräfte beteiligt.

(b) Auf beiden Seiten muss ein Mindestmaß an zentralgelenkter Organisation der Kriegführenden und des Kampfes gegeben sein.

\footnotetext{
5 Vgl. Gasser, Hans-Peter: Protection of the Civilian Population in: Fleck, Dieter (Hrsg.): The Handbook of Humanitarian Law in Armed Conflicts, Oxford 1999, S. $209 \mathrm{ff}$.

6 Vgl. Daase, Christopher: »Das humanitäre Völkerrecht und der Wandel des Krieges « in: Hasse, Jana; Müller, Erwin; Schneider, Patricia (Hrsg.): Humanitäres Völkerrecht, Baden-Baden 2001, S.132ff.

7 Der einzig relevante Unterschied meiner Definition zu der AKUF-Definition ist, dass ich nicht festlege, dass mindestens eine Seite staatlich sein muss. Auf die Anzahl der aktuellen Konflikte hat dies jedoch keine Auswirkungen.
} 
(c) Die bewaffneten Operationen ereignen sich mit einer gewissen Kontinuität und nicht nur als gelegentliche, spontane Zusammenstöße.

Alle anderen Formen der bewaffneten Auseinandersetzung sind keine Kriege. ${ }^{8}$ Diese Definition zugrundgelegt, fanden in den letzten zwölf Monaten in 19 Ländern Kriege statt. ${ }^{9}$ Einige kritische Fälle sind, obwohl die Kontinuität der Kampfhandlungen nicht immer gegeben ist, wie in Algerien, oder obwohl die nichtstaatliche Seite nicht unbedingt zentral gelenkt ist, wie in Thailand, mit enthalten. Andere kritische Fälle wie z.B. die bewaffneten Konflikte in Nigeria, Pakistan, Senegal, dem Jemen, Burundi, Haiti und Laos sind meist aufgrund der mangelnden Kontinuität der Kampfhandlungen oder des viel zu niedrigen Organisationsgrades der nichtstaatlichen Seite nicht mit aufgeführt. ${ }^{10}$

Zur besseren Veranschaulichung des hier Dargelegten habe ich alle Kriege auf einer Karte dargestellt und den Grad der Verletzung des humanitären Völkerrechts durch drei verschiedene Stufen wiedergegeben. Eine jede Kategorisierung hat sicherlich etwas Verkürzendes und Pauschalisierendes an sich, denn in diesem Zusammenhang gibt es keine absolute Trennschärfe. Als systematischer Überblick ist eine solche Kategorisierung meines Erachtens dennoch sinnvoll. Die Darstellung durch eine Karte für die staatliche Seite (Abb. 1) und eine andere Karte für die nichtstaatliche Seite (Abb.2) ermöglichen zusätzlich einen schnellen Gesamtüberblick der unterschiedlichen Akteursseiten. Jeder Krieg wird im Folgenden kurz dargestellt.

Die von mir gewählten Stufen sind folgende:

Stufe I (hellgrau): Die Verletzungen des Art. 3 treten, wenn überhaupt, nur in einem sehr geringen Ausmaße auf. Bei Verletzungen versucht die jeweilige Seite die Verantwortlichen zur Rechenschaft zu ziehen. Es gibt keine systematische Strategie, die die Verletzung des humanitären Völkerrechts explizit mit einbezieht.

Stufe II (dunkelgrau): Die Verletzungen des Art. 3 treten vermehrt auf und werden selten oder gar nicht von der Konfliktpartei unterbunden. Teilweise sind Strategien erkennbar, die die Verletzung des humanitären Völkerrechts explizit beinhalten.

Stufe III (schwarz): Die Verletzungen des Art. 3 treten massiv auf. Die Kombattanten werden angehalten, Handlungen, die Art. 3 verletzen, als militärische Strategie zu vollziehen. Für die Verletzungen wird niemand zur Rechenschaft gezogen.

8 Als bewaffnete Konflikte werden deshalb gewaltsame Auseinandersetzungen bezeichnet, bei denen die Kriterien der Kriegsdefinition nicht in vollem Umfang erfüllt sind. In der Regel handelt es sich dabei um Fälle, in denen eine hinreichende Kontinuität der Kampfhandlungen nicht mehr oder noch nicht gegeben ist.

9 In einigen Ländern wie Kolumbien, Indien und den Philippinen finden sogar mehrere Kriege gleichzeitig statt.

10 Für eine ausführliche Darstellung der Kriege und bewaffneten Konflikte vgl. Schreiber, Wolfgang (Hrsg.): Das Kriegsgeschehen 2005, Wiesbaden 2006 und ders. (Hrsg.): Das Kriegsgeschehen 2006, Wiesbaden 2007. Vgl. zur Übersicht ferner die einschlägigen Informationen auf der homepage der Arbeitsgemeinschaft Kriegsursachenforschung (http://www.sozialwiss. uni-hamburg.de/publish/Ipw/Akuf/index.htm).

\subsection{Lateinamerika}

Nachdem Lateinamerika noch bis in die 1990er Jahre von insgesamt 30 Kriegen u.a. in Nicaragua, Chile und Guatemala einer der zentralen Kriegsschauplätze weltweit war, hat sich die Region inzwischen weitestgehend beruhigt. Allein in Kolumbien stehen sich weiterhin Rebellen, rechtsgerichtete Paramilitärs und die Armee in einem blutigen Bürgerkrieg gegenüber.

\section{Kolumbien}

In dem bereits 1964/65 begonnenen Krieg zwischen FARC/ ELN und der kolumbianischen Regierung bekämpft die staatliche Seite nicht nur die FARC/ELN, sondern in den letzten Jahren auch die rechtsgerichteten paramilitärischen Einheiten (AUC). Immer wieder werden dabei auch zivile Ziele in den von den Rebellen kontrollierten Gebieten angegriffen. Festgenommene Rebellen werden rechtskräftig verurteilt und inhaftiert. Immer wieder wird über eine unverhältnismäßige Härte von Seiten der Polizei und des Militärs berichtet. Die jeweiligen Menschenrechtsverletzungen durch Armee und Polizei werden so gut wie nie strafrechtlich geahndet. Dennoch ist keine allgemeine systematische Verletzung des humanitären Völkerrechts zu konstatieren, da die Regierung Kolumbiens sich in den vergangenen Jahren bemüht hat, die Verletzungen des humanitären Völkerrechts seitens staatlicher Akteure zu reduzieren und die ehemals von ihnen unterstützen paramilitärischen Einheiten der AUC zu entwaffnen. Anders verhalten sich jedoch FARC/ELN und die AUC. Inzwischen kämpfen alle nichtstaatlichen Einheiten gegeneinander um die Kontrolle des Drogenanbaus und Drogenhandels. Dabei sind gezielte Tötungen unliebsamer Politiker, Menschenrechtler und Gewerkschafter an der Tagesordnung. Auch werden immer wieder Zivilisten entführt, um Lösegeld zu erpressen oder von ganzen Dörfern systematisch Schutzgeld erpresst.

\subsection{Afrika}

Afrika ist weiterhin ein zentraler Schauplatz des weltweiten Kriegsgeschehens. Zwar ist Westafrika inzwischen frei von Kriegen, doch sind besonders die Länder Zentralafrikas Austragungsort verschiedener Bürgerkriege. Der Krieg in der sudanesischen Region Darfur beeinflusst inzwischen auch die angrenzenden Staaten Tschad und Zentralafrikanische Republik. Im Tschad hat sich daraus inzwischen ein eigener Krieg entwickelt. Der Krieg im Osten des Kongo konnte auch nach den Wahlen nicht beendet werden, und der Krieg in Somalia rückte durch den Einmarsch der äthiopischen Truppen in diesem Jahr besonders in das Blickfeld der Weltöffentlichkeit.

\section{Sudan}

In dem Krieg, der hauptsächlich in der im Westen liegenden Region Darfur ausgetragen wird, kämpfen verschiedene von der Regierung unterstützte arabische Reitermilizen (Dschandschawid) und die Regierung gegen die lokalen schwarzafri- 
Abb. 1: Verletzungen des Art. 3 der Genfer Konvention - staatliche Akteure

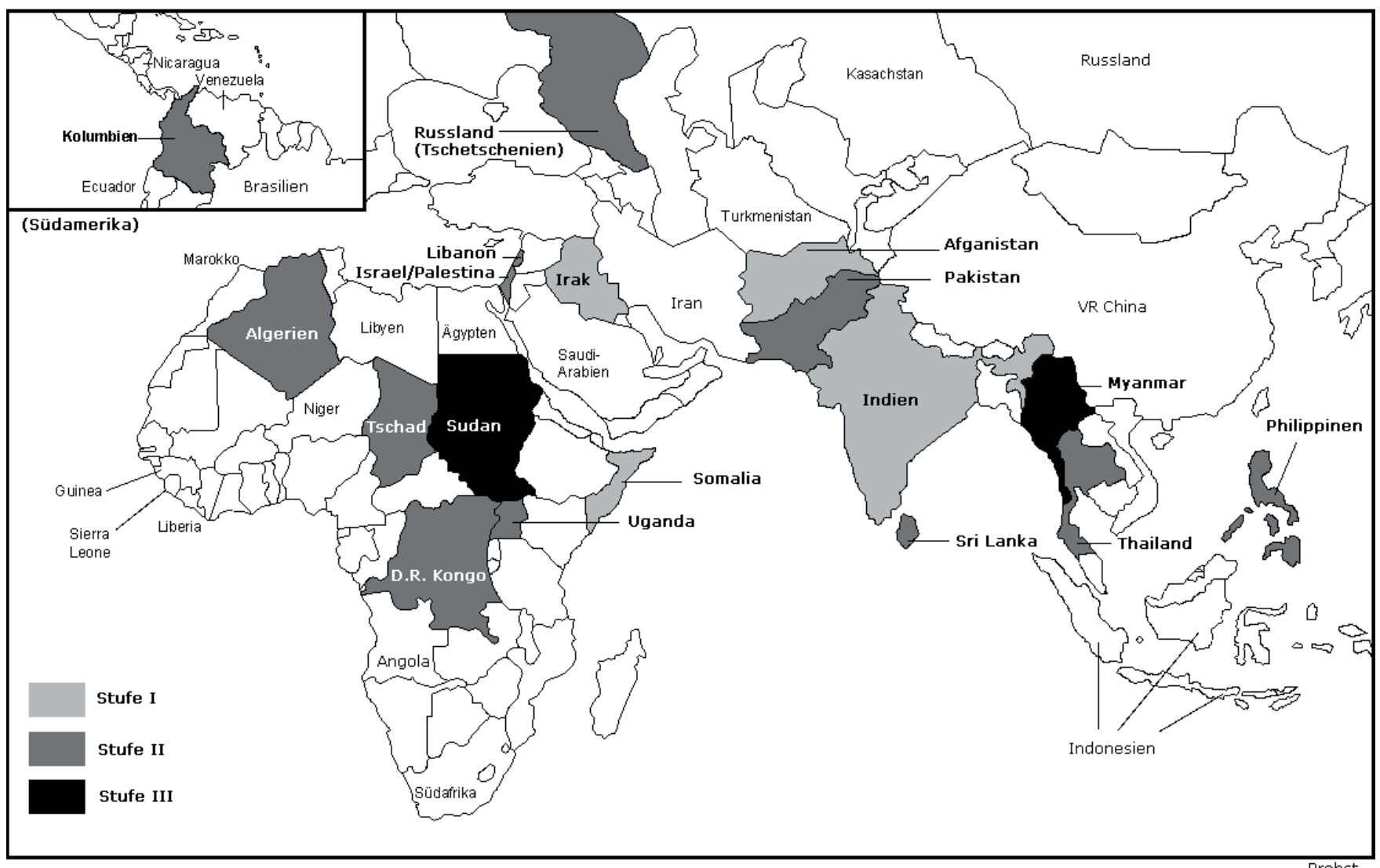

Abb. 2: Verletzungen des Art. 3 der Genfer Konvention - nichtstaatliche Akteure

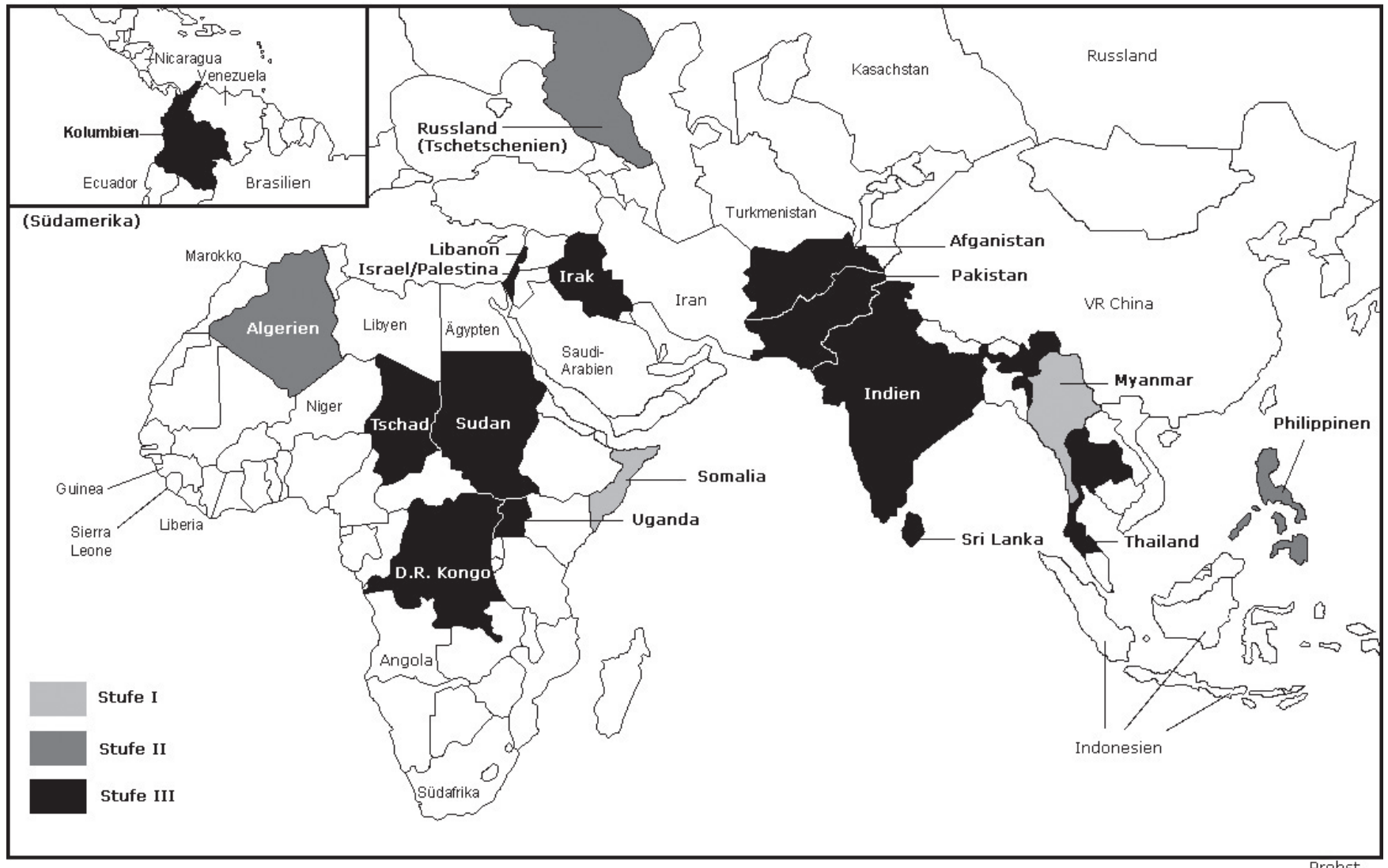


kanischen Rebellengruppen wie SLA und JEM. Aber auch die Rebellengruppen bekämpfen sich inzwischen gegenseitig. Die sudanesische Regierung setzt bei ihrem Kampf gegen die Rebellen trotz verschiedener Friedensinitiativen auf eine systematische Vertreibung der in Darfur lebenden schwarzafrikanischen Bauern. Dörfer werden von der Luftwaffe bombardiert, während die Reitermilizen andere Dörfer angreifen und deren Zivilbevölkerung vertreiben oder ermorden. Auch Massenvergewaltigungen werden immer wieder verübt. Die Rebellen der JEM und SLA versuchen diese Angriffe abzuwehren, verüben aber auch selbst systematische Angriffe auf die arabische Zivilbevölkerung, bei denen ebenfalls Zivilisten vertrieben oder ermordet werden. Zwar wurde im Mai 2006 mit dem Darfur Peace Agreement ein Friedensabkommen zwischen Regierung und einigen Rebellenfraktionen geschlossen, jedoch von keiner Seite eingehalten. Insgesamt sollen bisher über 200.000 Zivilisten getötet worden und zwei Millionen weitere geflohen sein.

\section{Tschad}

Der Krieg im Tschad ist im letzten Jahr maßgeblich durch das Kriegsgeschehen im Sudan mitbestimmt worden. Immer wieder dringen Verbände von arabischen Reitermilizen (Dschandschawid) in die Flüchtlingslager für die aus Darfur Vertriebenen ein und ermorden unzählige Zivilisten. Auch haben sich inzwischen eigene arabische Reitermilizen und Rebellengruppen im Tschad organisiert, die systematisch die Dörfer der lokalen schwarzafrikanischen Zivilbevölkerung angreifen. Aus den Flüchtlingslagern rekrutieren sich zusätzlich die Kämpfer der schwarzafrikanischen Rebellengruppen, die wiederum die arabische Zivilbevölkerung angreifen. Mehrmals haben arabische Rebellen versucht, die Hauptstadt N'Djamena anzugreifen und damit die Regierungsmacht zu erlangen. Nur mit Hilfe der französischen Armee konnten die Angriffe der Rebellen zurückgeschlagen werden. Die Regierung geht sehr hart gegen all jene vor, die im Verdacht stehen, die Rebellen zu unterstützen. Verdächtige werden gefoltert. Exekutionen finden auch ohne Gerichtsverhandlung statt.

\section{Kongo}

Verschiedene bewaffnete Gruppen, die bisher nicht in die regulären Streitkräfte integriert werden konnten, verüben fast täglich schwere Verbrechen gegen die Zivilbevölkerung, vor allem in den ostkongolesischen Provinzen Kivu und Ituri. Tausende Zivilisten wurden im vergangenen Jahr vergewaltigt, entführt oder ermordet. Insgesamt sollen noch über 11.000 Kinder Mitglieder der verschiedenen Rebellenarmeen sein. Viele der Rebellengruppen stammen aus Ruanda und Uganda, und auch deshalb sind in der Vergangenheit ruandische und ugandische Armeeverbände in diese Gebiete eingedrungen. Die regulären Streitkräfte aus Uganda, Ruanda und dem Kongo sind aber ebenso für massive Verletzungen des humanitären Völkerrechts verantwortlich. Die Regierung des Kongo versucht jedoch, diesem Verhalten Einhalt zu gebieten. Besonders im letzten Jahr wurden, sicherlich auch aufgrund der internationalen Präsenz der UN-Friedenstruppe, wiederholt Soldaten wegen massiver Verbrechen gegen die Menschlichkeit angeklagt und auch verurteilt.

\section{Uganda}

Das Kriegsgeschehen in Uganda wird durch den Kampf zwischen der Armee und der christlich-fundamentalistischen Lords Resistance Army (LRA) bestimmt. Dabei zeichnet sich die LRA durch eine hohe Anzahl von Kindersoldaten aus. Massenvergewaltigungen, gezielte Ermordungen von Zivilisten und flächendeckende Vertreibungen durch die LRA haben dazu geführt, dass inzwischen mehr als eine Million Menschen in Uganda selbst intern vertrieben sind. Die Regierungssoldaten haben in der Vergangenheit mehrfach Verdächtige und gefangen genommene Mitglieder der LRA gefoltert. Es herrscht weiterhin eine hohe Disparität zwischen den Berichten über Folter und den darauf folgenden Verurteilungen. Momentan werden Friedensverhandlungen geführt. Die Regierung hat mit 16 Jahren Verspätung die UN-Konvention gegen Folter unterzeichnet. Der vor den Friedensverhandlungen ausgehandelte Waffenstillstand lief jedoch am 1. März 2007 aus und wurde nicht verlängert.

\section{Somalia}

Nachdem die Koalition aus verschiedenen islamischen Rebellengruppen (UIC) im vergangenen Jahr Mogadischu erobern konnte und daraufhin immer näher an den Sitz der Übergangsregierung in Baidoa vordrang, gelang es den Kräften der Übergangsregierung zusammen mit der äthiopischen Armee, die Rebellen zurückzudrängen und im Dezember 2006 Mogadischu zu erobern. In den darauf folgenden Tagen konnte die UIC gänzlich von somalischem Territorium zurückgedrängt werden. Einige Rebellenführer kündigten daraufhin die Aufnahme einer Guerillataktik an. Sowohl von Seiten der Rebellen als auch von Seiten der regulären Truppen der Übergangsregierung und der äthiopischen Armee sind nur wenige Verletzungen des humanitären Völkerrechts bekannt geworden. Viele Zivilisten flohen rechtzeitig aus den umkämpften Gebieten, kehrten aber nach Beendigung der Auseinandersetzungen zurück. In der Regel versuchten beide Seiten, die ansässigen Zivilisten nicht direkt zu treffen. Diese Lage kann sich jedoch sehr schnell mit der Ankunft der bis zu 8.000 Soldaten starken Friedenstruppe der Afrikanischen Union (AU) ändern, da viele der Anführer der UIC einen breiten militärischen Widerstand gegen jeden ausländischen Soldaten auf somalischem Territorium angekündigt haben.

\section{Algerien}

Trotz mehrerer Friedensinitiativen und eines 2005 verabschiedeten Amnestiegesetzes für ehemalige Rebellen dauern die Kämpfe zwischen der Armee und mindestens 500 militanten Islamisten, insbesondere der Salafistischen Gruppe für Predigt und Kampf (GSPC) weiter an. Im letzten Jahr wurden über 300 Menschen durch direkte Kampfhandlungen getötet. 
Zwar waren unter den Getöteten auch einige Zivilisten, doch kann man hier nicht von systematischen Angriffen auf die Zivilbevölkerung von einer der beiden Seiten sprechen. Die besonders in den 1990er Jahren durch die Sicherheitskräfte verübten Morde, Entführungen und Folterungen sind inzwischen sehr selten geworden. Strafrechtliche Verfolgung von etwaigen Verletzungen gibt es allerdings nicht. Die islamistischen Rebellen entführen immer wieder Touristen oder andere Zivilisten, um Lösegeld oder die Freilassung von gefangenen Mitkämpfern zu erpressen.

\subsection{Vorderer und Mittlerer Orient/Zentralasien/ Ostasien}

Das Geschehen wird momentan von den Kriegen im Libanon, in Israel, Irak und Afghanistan bestimmt. Aber auch die seit langem andauernden Kriege in den mehrheitlich kurdischen Gebieten der Türkei sowie in der russischen Kaukasusrepublik Tschetschenien sind nicht beendet.

\section{Irak}

Der Krieg im Irak wird durch den Kampf zwischen den von den USA dominierten multinationalen Truppen bzw. der irakischen Armee auf der einen und verschiedenen schiitischen und sunnitischen Rebellen bzw. Terrororganisationen auf der anderen Seite bestimmt. Zusätzlich bekämpfen sich die sunnitischen und schiitischen Gruppen vermehrt gegenseitig. Massive direkte Angriffe auf Zivilisten der jeweils anderen Ethnie oder Religion seitens der Rebellen sind an der Tagesordnung. Wiederholte Entführungen und Ermordungen von irakischen und westlichen Zivilisten bestimmen den Alltag. An manchen Stellen sind politisch motivierte Gewalt und Kriminalität nicht mehr voneinander zu trennen. Die staatlichen Einheiten sind in der Vergangenheit nicht immer mit der nötigen Vorsicht vorgegangen, um Zivilisten zu schonen. Fälle von Folter durch irakische und amerikanische Truppen sind keine Seltenheit, in der Regel werden diese jedoch geahndet und die Verantwortlichen vor Gericht gestellt. Immer wieder werden Menschen auch ohne Verhandlung eingesperrt. Insgesamt muss man aber festhalten, dass besonders die multinationalen Truppen versuchen, die zivilen Opfer der kriegerischen Auseinandersetzung so niedrig wie möglich zu halten und im Allgemeinen das humanitäre Völkerrecht zu achten.

\section{Türkei}

Die für ein unabhängiges Kurdistan kämpfenden Rebellen der PKK lieferten sich im vergangenen Jahr immer wieder intensive Gefechte mit der türkischen Armee, vor allem im Osten des Landes. Die türkische Armee setzt bei dem Versuch, die Gebiete zu kontrollieren, massive Zwangsmaßnahmen gegenüber der kurdischen Zivilbevölkerung, inklusive wahllosen Festnahmen, Folter und Vertreibungen ein. Die Türkei bemühte sich jedoch in den vergangenen Jahren, die Übergriffe auf die Zivilbevölkerung einzudämmen. Bisher sind noch keine ranghö- heren Militärs wegen Menschenrechtsverletzungen angeklagt worden, jedoch wurden einige Polizisten vor Gericht gestellt und auch verurteilt. Die Rebellen ihrerseits verüben immer wieder Bombenanschläge auf zivile Ziele. Schutzgelderpressungen sind in den von den Rebellen kontrollierten Gebieten üblich. Auch werden immer wieder unliebsame Politiker und zivilgesellschaftliche Akteure gezielt ermordet. Zum 1. Oktober 2006 wurde von der PKK einseitig ein Waffenstillstand ausgerufen, der aber bisher von der türkischen Seite nicht beachtet wurde.

\section{Afghanistan}

Der Krieg zwischen den durch die USA geführten Truppen der Operation »Enduring Freedom « sowie der neu aufgebauten afghanischen Armee und den Taliban hat sich in den letzten Monaten weiter intensiviert. Besonders die südlichen an Pakistan grenzenden Provinzen sind Austragungsort der Gefechte. Viele der Taliban haben inzwischen auf pakistanischem Gebiet verschiedene Lager aufgebaut, sie werden dort aber auch vom pakistanischen Militär angegriffen. In den letzten zwölf Monaten wurden weit über 100 Selbstmordanschläge von Rebellen in Afghanistan verübt. Allein 2006 wurden ungefähr 4.000 Menschen getötet, davon 1.000 Zivilisten. Unter den Getöteten waren viele Wahlhelfer, Kleriker und andere Hilfskräfte. Die afghanischen Armee- und Polizeikräfte nahmen in der Vergangenheit hunderte Verdächtige ohne Anklage fest. Seitens der Internationalen Truppen sind nur wenige Verletzungen des humanitären Völkerrechts bekannt geworden und in allen Fällen wurden die Täter angeklagt. Weitere gewaltsame Auseinandersetzungen insbesondere zwischen Privatarmeen verfeindeter Warlords forderten zusätzlich etliche zivile Todesopfer.

\section{Pakistan}

Neben dem Konflikt zwischen der pakistanischen Armee und den aus Afghanistan geflüchteten Taliban wird der Krieg in Pakistan hauptsächlich durch die Kämpfe der Regierungsarmee gegen tribale Rebellen aus der Provinz Belutschistan bestimmt. In einer großen Militäroffensive am 26. August 2006 versuchte die Regierung, die Rebellen um den Klanführer Nawab Akbar Bugti zu besiegen. Bei dieser Offensive wurde Bugti getötet und die Rebellen massiv geschwächt. Das pakistanische Militär setzt bei Angriffen auch immer wieder die Luftwaffe ein, die Stellungen der Rebellen bombardiert, dabei aber auch wiederholt Zivilisten getroffen hat. Viele Verdächtige werden von Polizei oder Armee ohne Verhandlungen inhaftiert oder manchmal auch exekutiert. Die Rebellen versuchen ihrerseits, die Regierung Musharraf durch Bombenanschläge und Selbstmordattentate im gesamten Land zu destabilisieren. Die Frequenz der Anschläge hat sich nach dem Tod Bugtis noch einmal erhöht. Nicht immer ist dabei klar, ob Taliban oder Klankämpfer für die Anschläge verantwortlich sind. Insgesamt sind bei solchen Angriffen in den vergangenen zwölf Monaten über 400 Zivilisten getötet worden. Zusätzlich scheint der Konflikt zwischen Schiiten und Sunniten nicht mehr nur im Irak, sondern inzwischen auch in Pakistan gewaltsam ausgetragen zu werden. 


\section{Russland (Tschetschenien)}

Der Krieg zwischen der russischen Armee und tschetschenischen Unabhängigkeitskämpfern hat in den letzten Jahren an Intensität verloren. Die Rebellen wurden durch massive Angriffe der russischen Armee weit in die tschetschenischen Bergregionen zurückgedrängt. Inzwischen sind die Selbstmordanschläge tschetschenischer Rebellen im gesamten russischen Staatsgebiet deutlich zurückgegangen und, da sich die Hauptkampfregion inzwischen in wenig bevölkertem Gebiet befindet, sind auch immer weniger Zivilisten betroffen. Dennoch setzt die russische Armee weiterhin massive Zwangsmittel wie Exekutionen und Folter zur Bekämpfung der Gegenseite ein. Bei Angriffen der Rebellen auf Repräsentanten der von Russland eingesetzten tschetschenischen Autonomieregierung werden immer wieder auch Zivilisten getötet.

\section{Libanon}

Nachdem am 12. Juli 2006 bei einer Aktion der schiitischen Hisbollah-Miliz aus dem Süden des Libanon zwei israelische Grenzsoldaten entführt worden waren, griff das israelische Militär den Süden des Landes zunächst mit der Luftwaffe und dann mit Bodentruppen an. Der über einen Monat andauernde Krieg konnte erst durch einen UN-Friedensplan, der die Entsendung einer UN-Friedenstruppe in den Süden des Libanon vorsah, beendet werden. Bei dem Angriff der israelischen Armee versuchte diese zwar in der Regel, Zivilisten zu verschonen, indem sie diese aufforderte, die jeweiligen Kampfgebiete rechtzeitig zu verlassen, doch wurden mehrfach zivile Ziele durch israelische Luftangriffe getroffen. Man kann auf israelischer Seite nicht von einer gezielten Strategie, Zivilisten zu töten, sprechen, allerdings wurde durch die Art und Weise der Kriegsführung eine hohe Anzahl ziviler Opfer billigend in Kauf genommen. Die Hisbollah reagierte auf den Angriff, neben dem bewaffneten Widerstand gegen die israelischen Bodentruppen, mit dem Abschuss von mehreren tausend Katyusha-Raketen auf israelisches Territorium. Ziel war es dabei, möglichst viele israelische Zivilisten zu töten.

\section{Israel/Palästina}

Der Krieg zwischen der israelischen Armee und den verschiedenen palästinensischen Rebellengruppen weitete sich kurzzeitig aus, nachdem im Juli 2006 ein israelischer Soldat entführt worden war. Die israelische Armee drang daraufhin in den zuvor von israelischen Truppen und Siedlern geräumten Gazastreifen ein. Bei diesem erfolglosen Befreiungsversuch wurden über 250 Menschen getötet und fast 1.000 verletzt. Zusätzlich versuchte Israel, mutmaßliche Terroristen durch gezielte Raketenangriffe zu töten. Wiederholt wurden dabei auch Zivilisten getötet. Die Rebellengruppen schossen immer wieder Raketen auf israelische Siedlungen. Durch diese Angriffe starben im vergangenen Jahr über 40 israelische Zivilisten. Auch die Kämpfe zwischen den rivalisierenden palästinensischen Fraktionen Hamas und PLO eskalierten und forderten viele zivile Opfer. Gegenseitige Entführungen und Exekutionen von
Zivilisten waren an der Tagesordnung. Erst nach der Bildung einer Koalitionsregierung zwischen den beiden Fraktionen im Februar 2007 beruhigte sich die Lage allmählich.

Die Kriege in Asien konzentrieren sich seit vielen Jahren auf die Regionen Süd- und Süd-Ost Asien. Besonders Indien, Sri Lanka und Myanmar sind Kriegsregionen, aber auch die Kriege auf den Philippinen und im Süden Thailands sind nicht beendet.

\section{Indien}

Die Kriege in Indien werden durch unterschiedliche Konfliktdynamiken bestimmt. Im Nordosten kämpfen seit 1990 von Pakistan aus unterstützte Rebellengruppen für eine Unabhängigkeit des mehrheitlich muslimischen Kaschmir vom mehrheitlich hinduistischen Indien. Dieser Konflikt hat auch wiederholt zu Grenzkriegen zwischen Pakistan und Indien geführt. Der indische Staat geht in Kaschmir zwar mit äußerster Härte gegen die Rebellen vor, Zivilisten werden dabei aber nur sehr selten verletzt. Ziel der Militär- und Polizeieinsätze sind fast immer Kämpfer der verschiedenen Rebellengruppen. Bei etwaigem Fehlverhalten werden die Verantwortlichen oft von der indischen Regierung zur Rechenschaft gezogen. Ganz anders verhalten sich jedoch die verschiedenen Rebellengruppen der Region. Immer wieder werden Bomben gezündet, die direkt Zivilisten zum Ziel haben. Hier ist es offensichtlich Teil der militärischen Strategie, möglichst viele Zivilisten zu töten, um den indischen Staat zum Einlenken zu zwingen. Nicht grundsätzlich anders verhält es sich mit den im Nordosten des Landes ausgetragenen Kriegen zwischen der Armee und verschiedenen Befreiungsbewegungen der ethnischen Minderheiten aus Assam, Bodos, Nagaland und Tripura. Besonders die ULFA aus Assam (Manipur) greifen immer wieder meist bengalische Zuwanderer an. Dabei werden durch Bomben- und andere Angriffe Zivilisten direkt attackiert und getötet. Die indische Regierung versucht den Rebellen entgegenzuwirken, indem sie eine lokale Polizeimacht aufbaut. Verletzungen des humanitären Völkerrechts werden in diesem Zusammenhang nur sehr selten verübt. Zusätzlich kämpfen maoistisch orientierte Naxaliten im Zentrum Indiens gegen die Zentralregierung. Die Naxaliten erpressen im großen Umfang Schutzgeld von der Zivilbevölkerung, töten gezielt politische Oppositionelle und verüben immer wieder Überfälle auf Busse und Züge, welche die von ihnen kontrollierten Gebiete durchqueren.

\section{Sri Lanka}

Die schleppende Umsetzung des Friedensvertrages von 2004 und massive Streitigkeiten über die Verteilung der Hilfsgelder nach dem verheerenden Tsunami Ende 2004 führten dazu, dass der Konflikt zwischen der tamilischen LTTE und der singhalesischen Zentralegierung ab Ende 2005 erneut zum Krieg eskalierte. In den von der LTTE kontrollierten Gebieten erhebt diese Steuern und verwaltet die Gebiete eigenständig. Zusätzlich rekrutierte die LTTE gewaltsam Kinder für ihre Armee. Neben verschiedenen Vertretern der Regierung werden 
immer wieder tamilische Friedensaktivisten und gemäßigte Politiker gezielt von der LTTE getötet. Wegen einer Abspaltung der Rebellen unter Colonel Karuna bekämpfen sich die Rebellen inzwischen gegenseitig mit äußerster Brutalität. Die Regierungsseite reagierte auf die Angriffe der LTTE mit einer Bodenoffensive im Osten des Landes. Die Versorgungswege in die Rebellengebiete sind dauerhaft unterbrochen worden; hunderttausende Tamilen befinden sich auf der Flucht. Folterungen von Verdächtigen durch die Polizei werden immer wieder durchgeführt und in der Regel nicht angeklagt.

\section{Myanmar}

Der Konflikt zwischen der Zentralregierung von Myanmar und verschiedenen Rebellengruppen der unterschiedlichen ethnischen Minderheiten begann direkt nach der Unabhängigkeit des Landes 1948. Die durch die britische Kolonialherrschaft in den Kolonialstaat Burma integrierten ethnischen Minderheiten versuchen seit der Unabhängigkeit des Landes regionale Autonomie oder Unabhängigkeit gegenüber der burmesischen Bevölkerungsmehrheit durchzusetzen. Die Zentralregierung reagiert seit dem ersten Militärputsch 1962 auf jedwede Form von Autonomiebestrebungen mit massiver Härte. Ethnische Minderheiten werden aus den von ihnen besiedelten Gebieten vertrieben, Gefangene gefoltert und ohne Gerichtsprozess hingerichtet, Frauen immer wieder durch Streitkräfte vergewaltigt und brutal misshandelt. Selbst die größte momentan relevante Rebellenarmee, die Karen National Union (KNU), hat diesem Vorgehen wenig entgegenzusetzen und konzentriert sich inzwischen nur noch darauf, die eigene Bevölkerung vor den Übergriffen zu schützen. Um die Verluste unter den eigenen Kämpfern auszugleichen, rekrutierte die KNU in den letzten Jahren vermehrt Jugendliche in ihre Armee.

\section{Thailand}

Der Krieg in den südlichen Provinzen Thailands zeichnet sich durch eine extreme Asymmetrie zwischen muslimischen Rebellengruppen und den staatlichen Kräften aus. Während die Rebellengruppen sich fast ausschließlich auf Angriffe gegen meist buddhistische Zivilisten konzentrieren und hin und wieder aus dem Hinterhalt die Sicherheitskräfte angreifen, versuchen Polizei und Armee der sich immer weiter verschlechternden Sicherheitslage durch Ausgangssperren und Straßenkontrollen Herr zu werden. Direkte Kampfhandlungen zwischen den beiden Seiten sind selten. Während es bei den Rebellen strategisches Mittel ist, mit Bombenanschlägen und Überfällen möglichst viele unschuldige Zivilisten zu töten, versuchen die Sicherheitskräfte durch umfangreiche und oft fast wahllose Festnahmen, bei denen die Gefangenen auch gefoltert werden, an die Kommandeure der jeweiligen Rebellengruppen zu gelangen. Insgesamt wurden im letzten Jahr mindestens 1.500 Zivilisten getötet und über 2.000 verletzt.

\section{Philippinen}

Das Kriegsgeschehen auf den Philippinen wird durch zwei unterschiedliche Konflikte bestimmt: Zum einen kämpfen kommunistische Rebellen der New People's Army (NPA) besonders auf den nördlichen Inseln des Landes gegen die Regierung. Zum anderen kämpfen muslimische Rebellen verschiedener Gruppen (Abu Sayyaf, Moro Islamic Liberation Front) auf den muslimischen Inseln im Süden des Landes für mehr Autonomie oder Unabhängigkeit. Bei dem Versuch, die NPA und die muslimischen Rebellen zu bekämpfen, wird immer wieder darüber berichtet, dass Zivilisten von der Regierung wahllos festgenommen werden oder einfach verschwinden. Folter und auch Exekutionen ohne Gerichtsverhandlung sind dabei keine Seltenheit. Zusätzlich werden wiederholt angebliche Sympathisanten der Rebellen, Gewerkschafter und linke Politiker durch Unbekannte erschossen. Eine Untersuchung der UN bestätigte, dass die Sicherheitskräfte für mindestens 832 dieser Übergriffe seit 2001 verantwortlich sind. Zwar gibt es keine Hinweise darauf, dass die Regierung selbst diese direkt angeordnet hat, doch werden nur sehr selten Polizisten oder Armeeangehörige für Ermordungen und Entführungen vor philippinischen Gerichten angeklagt und verurteilt. Neben den direkten Angriffen auf Regierungstruppen entführen die Rebellen Zivilisten und töten gezielt Politiker. Inzwischen versucht die von der UN unterstützte Joint Monitoring Mission, der auch NPA und Regierung angehören, gemeinsam Verstöße beider Seiten gegen die Menschenrechte und das humanitäre Völkerrecht zu überwachen.

\section{Fazit}

Anhand dieser Darstellung der Verletzungen des humanitären Völkerrechts im aktuellen Kriegsgeschehen lässt sich eindeutig festhalten, dass besonders nichtstaatliche Akteure massiv das humanitäre Völkerrecht verletzen. Ausnahmen stellen hier nur die islamistischen Rebellen in Somalia und die sich aus ethnischen Minderheiten zusammensetzenden Rebellen in Myanmar dar. Auf staatlicher Seite bemühen sich fast alle Akteure, das humanitäre Völkerrecht einzuhalten. Ausnahmen sind hier die international schon wiederholt geächteten Regime im Sudan und in Myanmar. Gründe dafür sind natürlich vielschichtig: Zum einen bringt die Asymmetrie der Kriegsführung nichtstaatliche Akteure dazu, alle ihnen zur Verfügung stehenden Zwangsmittel auszuschöpfen. Zum anderen gilt, dass die staatliche Seite viel intensiver in die internationale Gemeinschaft eingebunden und häufig auch massiv von Gebernationen finanziell, wirtschaftlich und politisch abhängig ist. Damit stehen Staaten unter einem ganz anderen Rechtfertigungsdruck. Diese These wird zusätzlich dadurch gestützt, dass diejenigen Staaten, die massive Verletzungen des humanitären Völkerrechts begehen, international im Abseits stehen. Wenn dies der Fall ist, würde dadurch das Argument verstärkt werden, dass rechtliche Rahmenbedingungen und internationale Verträge, auch bei bisher mangelnden Sanktionsmöglichkeiten, positive Auswirkungen auf die Handlungen der Akteure haben. 